\title{
Density functional theory for random sequential adsorption*
}

\author{
Matthias Schmidt \\ Institut für Theoretische Physik II, Heinrich-Heine Universität Düsseldorf, Universitätsstraße 1, \\ D-40225 Düsseldorf, Germany \\ E-mail: mschmidt@thphy.uni-duesseldorf.de
}

Received 21 June 2002, in final form 29 August 2002

Published 8 November 2002

Online at stacks.iop.org/JPhysCM/14/12119

\begin{abstract}
We treat the non-equilibrium process of random sequential adsorption of hard particles onto a solid substrate by means of a geometry-based density functional theory. As a prerequisite we solve the zero-dimensional case exactly and use it to construct density functionals in higher dimensions, permitting the treatment of adsorption onto arbitrary spatially inhomogeneous substrates. As applications we study the influence of a hard boundary of the adsorption region in the one-dimensional car-parking problem and for colloidal deposition on a twodimensional solid substrate. Comparing to our computer simulation results, we find that the respective density functionals correctly predict the oscillatory density profiles near the boundary, with amplitudes that are considerably smaller than in the corresponding equilibrium models.
\end{abstract}

\section{Introduction}

Random sequential adsorption (RSA) is one process that allows the systematic study of structure emerging in non-equilibrium situations (see [1] for a classic work and [2,3] for reviews). In RSA a $d$-dimensional space (the substrate) is sequentially filled by particles. Each newly placed (deposited) particle is subject to the influence of the particles already present. In contrast, all deposited particles remain frozen at their positions and do not adjust to the presence of any newly deposited particles. Due to the intrinsic asymmetry of interactions, such a system is not characterized by an equilibrium distribution.

In the simplest case the particles are represented by hard spheres (HS), but generalizations exist; moreover RSA is often studied in low spatial dimensionality. In one dimension (1D) one refers to the (random) car-parking problem where exact results, e.g. for the pair correlation function [4], are known. In 2D, the RSA of HS is a common model for describing the adsorption of proteins [5, 6] or colloids [3] on solid surfaces.

* This work is dedicated to the memory of Yasha Rosenfeld. 
Standard equilibrium liquid-state methods have been extended to treat such systems [7]; one particular example is the Percus-Yevick integral equation for RSA [8]. The principal derivation relies on the replica trick for studying quenched-annealed systems. In a binary quenched-annealed system the quenched species is commonly used to model a porous material and the annealed species an adsorbate fluid. Extending this framework to multi-component systems and differentially quenching single species allows one to treat the RSA process, where in a certain limit each particle in RSA corresponds to one species in the differentially quenched system.

Within the framework of density functional theory (DFT) [9] the equilibrium systems that correspond to the above-mentioned RSA models (hard rods in 1D, hard discs in 2D) are prototype examples where in 1D Percus derived the exact free energy functional [10], and in higher dimensions, via Rosenfeld's fundamental-measure theory (FMT), excellent approximations are available [11-14].

Along a similar route to that above for integral equation theory, recently DFT was extended to quenched-annealed systems $[15,16]$. The 'trick' for arriving at an approximation for the excess free energy is to solve the statistical mechanics in an idealized strongly confined situation called the 0D limit exactly, and input this knowledge into the geometric concept of FMT.

In the present contribution we propose, to the best of our knowledge, the first DFT for RSA. It is based on the exact solution for RSA in the OD limit, and uses Rosenfeld's concept to generalize to higher dimensions. As test cases, we consider particle deposition near a hard wall in 1D and 2D. Good agreement of the theoretical density profiles with our computer simulation data is found. The density profiles show considerably less pronounced structure when compared to the corresponding equilibrium systems. We can trace that back to the relatively smaller contribution of the excess part of the free energy compared to the ideal part in the RSA case, a feature that is already present in 0D.

The paper is organized as follows. In section 2 we define the process of RSA on an inhomogeneous substrate more explicitly. Section 3 is devoted to the exact solution for RSA in OD and the subsequent DFT generalization to higher dimensions. In section 4 we use hard boundaries of the adsorption region in the 1D car-parking problem and for 2D colloidal deposition on a smooth wall as test cases to compare theoretical results to those from our computer simulations. We conclude in section 5.

\section{The model}

Inhomogeneous RSA is characterized by spheres with radius $R$ that are placed sequentially onto a $d$-dimensional space characterized by an external potential $V_{\text {ext }}(r)$ where $r$ is the spatial coordinate. The placement is done according to repeated application of the following procedure.

- A trial sphere is placed at a random position $r$.

- The trial sphere is removed with probability $1-\exp \left(-\beta V_{\mathrm{ext}}(\boldsymbol{r})\right)$, where $\beta$ is the inverse temperature.

- The trial sphere is removed if it overlaps with any other sphere. Thereby overlap is characterized by a separation of sphere centres less than $2 R$.

As a dimensionless density we use the coverage $\theta=v_{d} \rho$, where $\rho$ is the number density of particles and $v_{d}$ is the volume of a $d$-dimensional 'sphere', i.e. $v_{1}=2 R, v_{2}=\pi R^{2}$. The particle diameter is denoted by $\sigma R$. 


\section{Theory}

\subsection{Overview}

We seek to obtain the density distribution $\rho(\boldsymbol{r})$ for inhomogeneous RSA from a grand potential functional $\Omega[\rho(\boldsymbol{r})]$, which becomes minimal for the actual RSA profile. Hence the RSA profile fulfils the Euler-Lagrange equation $\delta \Omega / \delta \rho(\boldsymbol{r})=0$. We write the functional as a sum

$$
\Omega[\rho(\boldsymbol{r})]=F_{\mathrm{id}}[\rho(\boldsymbol{r})]+F_{\mathrm{exc}}[\rho(\boldsymbol{r})]+\int \mathrm{d} \boldsymbol{r} \rho(\boldsymbol{r})\left[V_{\mathrm{ext}}(\boldsymbol{r})-\mu\right],
$$

where $r$ is the $d$-dimensional space coordinate, $\mu$ is a Lagrange parameter used to adjust the overall density, $F_{\text {id }}$ is the ideal-gas free energy functional, and $F_{\text {exc }}$ is the contribution due to interactions with already adsorbed particles. The ideal-gas part is the same as in the equilibrium case and is given by

$$
\beta F_{\mathrm{id}}[\rho(\boldsymbol{r})]=\int \mathrm{d} \boldsymbol{r} \rho(\boldsymbol{r})\left[\ln \left(\Lambda^{d} \rho(\boldsymbol{r})\right)-1\right],
$$

where $\Lambda$ is the (irrelevant) thermal wavelength. In the following we will obtain an approximation for $F_{\text {exc }}$ using Rosenfeld's fundamental-measure concept, starting from the exact solution for RSA in zero dimensions.

\subsection{Zero dimensions}

The $0 \mathrm{D}$ limit is represented by a hard cavity that confines the particle centres to a small volume $v_{0 \mathrm{D}}$. Explicitly, we may chose a spherical cavity of radius $\epsilon$, such that $v_{0 \mathrm{D}}=4 \pi \epsilon^{3} / 3$, and it is assumed that $\epsilon \ll R$. Due to the HS interaction, the cavity may either be empty or be occupied by a single particle. Canonically this is trivial, but in the grand ensemble a non-trivial relation between average number of particles and chemical potential results. This can be used (see below) to construct density functionals (DF) in higher dimensions. In the following we deal with multi-component HS where $i=1, \ldots, m$ is an index that labels the species. This labelling will later permit description of RSA in terms of differential quenching. We first lay out the general procedure by reviewing the equilibrium case.

For an equilibrium $m$-component mixture of $\mathrm{HS}$ in $0 \mathrm{D}$, the mean number of particles of species $i$ is given as

$$
\bar{\eta}_{i}=\frac{z_{i}}{1+\sum_{j=1}^{m} z_{j}}
$$

where, for species $i$, the scaled fugacity is defined as $z_{i}=\exp \left(\beta \mu_{i}\right) v_{0 \mathrm{D}} / \Lambda_{i}^{3}$. Summing the individual contributions yields the total number of particles

$$
\bar{\eta}=\sum_{i=1}^{m} \bar{\eta}_{i}=\frac{z_{\mathrm{tot}}}{1+z_{\mathrm{tot}}},
$$

where we have defined the total fugacity $z_{\text {tot }}=\sum_{i=1}^{m} z_{i}$. Hence, not surprisingly, we recover the solution for one-component HS (without distinction of species) by setting $m=i=1$ in (3) and identifying $z_{1}$ in (3) with $z_{\text {tot }}$ in (4). The total fugacity is related to the total chemical potential via $\beta \mu_{\mathrm{tot}}=\ln \left(z_{\mathrm{tot}}\right)$, and the excess (over ideal gas) part is $\beta \mu_{\mathrm{ex}}=\beta \mu_{\mathrm{tot}}-\ln \bar{\eta}$, which is explicitly obtained from inversion of (4) as

$$
\beta \mu_{\mathrm{ex}}=-\ln (1-\bar{\eta}) \text {. }
$$


The relation to the $0 \mathrm{D}$ excess free energy $F_{0 \mathrm{D}}$ is via $\partial F_{0 \mathrm{D}} / \partial \bar{\eta}=\mu_{\mathrm{ex}}$. Integration yields

$$
\beta F_{0 \mathrm{D}}=(1-\bar{\eta}) \ln (1-\bar{\eta})+\bar{\eta} .
$$

From this result, DFs for equilibrium systems in higher dimensions can be derived (see sections $3.3,3.4)$.

We next perform the same steps as above for RSA. Particles are inserted into the cavity with increasing species index $i$, such that species $i$ is subject only to the influence of species $1, \ldots, i-1$. Later we will turn to the differentially quenched case by taking the proper limit of infinitely many components $m$ each at vanishing fugacity $z_{i}$. The mean number of particles of species $i$ is given by the relation

$$
\bar{\eta}_{i}=\frac{z_{i}}{\Pi_{j=1}^{i}\left(1+z_{j}\right)},
$$

differing from the equilibrium result (3). The total number of particles is obtained as

$$
\bar{\eta}=\sum_{i=1}^{m} \frac{z_{i}}{\Pi_{j=1}^{i}\left(1+z_{j}\right)} .
$$

In the following we assume all fugacities to be equal, $z \equiv z_{i}=z_{j}$ for all $i, j$. This simplifies the subsequent calculations but is not mandatory; we have checked that other choices (where $z_{i}$ is $i$-dependent) lead to the same final result after the RSA limit is taken. We can now simplify equation (8) and obtain

$$
\bar{\eta}=\sum_{i=1}^{m} \frac{z}{(1+z)^{i}}=1-\frac{1}{(1+z)^{m}} .
$$

In order to describe RSA a limiting procedure is required. We need to let $z \rightarrow 0$, to ensure that only a minute fraction of $i$-particles are present so that no interactions between particles of the same species $i$ occur. However, to arrive at non-vanishing $\bar{\eta}$ we let the number of components diverge, $m \rightarrow \infty$. Both limits are taken while keeping the total fugacity $z_{\text {tot }}=\sum_{i=1}^{m} z_{i}=m z$ constant. Performing the limit in (9) yields $\bar{\eta}=1-\exp \left(-z_{\text {tot }}\right)$, which can readily be inverted to $\beta \mu_{\text {tot }}=\ln (-\ln (1-\bar{\eta}))$. The excess part is

$$
\beta \mu_{\mathrm{ex}}=\ln (-\ln (1-\bar{\eta}))-\ln \bar{\eta},
$$

which can be integrated to give the OD excess free energy for RSA:

$$
F_{0 \mathrm{D}}=\operatorname{li}(1-\bar{\eta})-\gamma-(1-\bar{\eta}) \ln (-\ln (1-\bar{\eta}))-\bar{\eta}[\ln (\bar{\eta})-1],
$$

where $\gamma \approx 0.577216$ is Euler's constant, and $\operatorname{li}(x)=\int_{0}^{z} \mathrm{~d} t / \ln t$ is the logarithmic integral function. Note that the limiting procedure, $z \rightarrow 0, m \rightarrow \infty, m z=$ constant, can also be (trivially) applied in the equilibrium case (4) leading to an unchanged result (6).

It is interesting to compare (11) with the equilibrium result (6). Both free energies increase monotonically with $\bar{\eta}$, have positive curvature, and are defined for $0 \leqslant \bar{\eta} \leqslant 1$. We find, however, that the result for RSA is smaller by a factor of roughly $1 / 2$ over almost the entire density range; hence as a rule of thumb $F_{0 \mathrm{D}}^{\mathrm{RSA}} \approx F_{0 \mathrm{D}}^{\mathrm{eq}} / 2$. (Explicit numerical values, e.g. for $\bar{\eta}=0.7$, are $\beta F_{0 \mathrm{D}}^{\mathrm{RSA}}=0.1594, \beta F_{0 \mathrm{D}}^{\mathrm{eq}} / 2=0.3388 / 2=0.1694$.) As a consequence the ideal free energy (which is the same in both cases) gives a stronger contribution in RSA than in the equilibrium system. This property will persist through the construction of the DFs for higher dimensions, which we next turn to. 


\subsection{One dimension}

The excess (over ideal gas) free energy functional is expressed as

$$
\beta F_{\text {exc }}[\rho(x)]=\int \mathrm{d} x \Phi\left(\left\{n_{v}(x)\right\}\right),
$$

where the weighted densities $n_{v}(x)$ are obtained by $1 \mathrm{D}$ convolutions of the actual density profile $\rho(x)$ with weight functions $w_{\nu}$ :

$$
n_{v}(x)=\int \mathrm{d} x^{\prime} \rho\left(x^{\prime}\right) w_{v}\left(x-x^{\prime}\right), \quad v=0,1 .
$$

The weight functions are defined as

$$
\begin{aligned}
& w_{1}(x)=\Theta(R-|x|), \\
& w_{0}(x)=\delta(R-|x|) / 2,
\end{aligned}
$$

where $\Theta(r)$ is the step function, and $\delta(r)$ is the Dirac distribution. The excess free energy density is given by

$$
\Phi=n_{0} \beta F_{0 \mathrm{D}}^{\prime}\left(n_{1}\right) .
$$

where the prime denotes differentiation w.r.t. the argument. In the equilibrium case $\Phi=\Phi_{\mathrm{eq}}$, where we use (6) to obtain

$$
\Phi_{\mathrm{eq}}=-n_{0} \ln \left(1-n_{1}\right) \text {. }
$$

Up to now this is a reformulation of Percus's exact hard-rod functional [10]. For RSA we set $\Phi=\Phi_{\mathrm{RSA}}$ and usage of (11) gives

$$
\Phi_{\mathrm{RSA}}=n_{0}\left[\ln \left(-\ln \left(1-n_{1}\right)\right)-\ln n_{1}\right] .
$$

We emphasize that although this procedure generates the exact functional for the case of equilibrated hard rods, this does not hold in general; e.g. in the case of a 1D model colloidpolymer mixture [17] it yields bulk free energies differing from (albeit approximating well) the exact result [18].

\subsection{Two dimensions}

Again the excess free energy is a spatial integral:

$$
\beta F_{\mathrm{exc}}[\rho(\boldsymbol{r})]=\int \mathrm{d} \boldsymbol{x} \Phi\left(\left\{n_{v}(\boldsymbol{x})\right\}\right),
$$

and the weighted densities are obtained by $2 \mathrm{D}$ convolutions:

$$
n_{v}(\boldsymbol{x})=\int \mathrm{d} \boldsymbol{r} \rho(\boldsymbol{r}) w_{v}(\boldsymbol{x}-\boldsymbol{r}), \quad v=0,1, \mathrm{v} 1,2
$$

of the four 2D weight functions

$$
\begin{aligned}
& w_{2}(\boldsymbol{r})=\Theta(R-r), \\
& w_{1}(\boldsymbol{r})=\delta(R-r), \\
& \boldsymbol{w}_{\mathrm{v} 1}(\boldsymbol{r})=\delta(R-r) \boldsymbol{r} / r, \\
& w_{0}(\boldsymbol{r})=\delta(R-r) /(2 \pi R),
\end{aligned}
$$

where $r$ in equations (21)-(24) denotes the 2D position vector; the subscript $\mathrm{v}$ is a reminder that $\boldsymbol{w}_{\mathrm{v} 1}$ is a vector. The free energy density has the generic structure

$$
\Phi=n_{0} \beta F_{0 \mathrm{D}}^{\prime}\left(n_{1}\right)+\frac{n_{1} n_{1}-\boldsymbol{n}_{\mathrm{v} 1} \cdot \boldsymbol{n}_{\mathrm{v} 1}}{4 \pi} \beta F_{0 \mathrm{D}}^{\prime \prime}\left(n_{1}\right) .
$$


Inserting the equilibrium 0D excess free energy (6) yields the free energy density of Rosenfeld's functional in 2D:

$$
\Phi_{\mathrm{eq}}=-n_{0} \ln \left(1-n_{2}\right)+\frac{n_{1} n_{1}-\boldsymbol{n}_{\mathrm{v} 1} \cdot \boldsymbol{n}_{\mathrm{v} 1}}{4 \pi\left(1-n_{2}\right)} .
$$

For RSA we use (11) to obtain

$$
\Phi_{\mathrm{RSA}}=n_{0}\left[\ln \left(-\ln \left(1-n_{2}\right)\right)-\ln n_{2}\right]-\frac{n_{1} n_{1}-\boldsymbol{n}_{\mathrm{v} 1} \cdot \boldsymbol{n}_{\mathrm{v} 1}}{4 \pi}\left[\frac{1}{\left(1-n_{2}\right) \ln \left(1-n_{2}\right)}+\frac{1}{n_{2}}\right] .
$$

This completes the prescription for the $2 \mathrm{D}$ case. The generalization to $3 \mathrm{D}$ is straightforward, and also higher $d>3$ can be treated in principle [19].

\section{Results}

\subsection{One dimension}

Here and in the 2D case below, we study the influence of an external potential $V_{\text {ext }}$ that describes the boundary of the adsorption region, given by

$$
V_{\text {ext }}(x)= \begin{cases}\infty & x<R \\ 0 & \text { otherwise. }\end{cases}
$$

In order to provide benchmark results we have carried out computer simulations of the RSA process with fixed total number of insertion trials. We have stayed somewhat away from the jamming limit, where no further particles can be deposited due to hard-core overlap. In 1D this jamming coverage is $\theta=0.7476$, and we have considered $\theta=0.6883$, i.e. $\sim 92 \%$ of the jamming value.

In the simulations a second wall is used in order to build up a finite system. The resulting external potential is $V_{\text {ext }}(x)+V_{\text {ext }}(L-x)$, where the wall distance $L$ is chosen sufficiently large that the density profiles from both walls are well decoupled from each other; we use $L=20 \sigma$, which we find to be amply sufficient. The number of insertion trials is fixed to 100; hence the number of particles present in the system fluctuates from realization to realization. Averaging over $10^{6}$ realizations yields the above-quoted value for the coverage. Histograms of the particle coordinates are used to obtain $\rho(x)$.

Within DFT we numerically minimize (via iteration of the corresponding Euler-Lagrange equation) the grand potential functional $\Omega$, equation (1), where the excess part, $F_{\text {exc }}$, in the present $1 \mathrm{D}$ case is given in section 4.1. The Lagrange parameter $\mu$ is determined to yield the prescribed coverage.

Results from simulation and theory are displayed in figure 1. The density has a maximal value at contact with the wall, $x=R^{+}$. It decreases monotonically over a distance of one particle diameter $(\sigma / 2<x<3 \sigma / 2)$. At $x=3 \sigma / 2$ a kink occurs, and for larger $x$ the oscillations are rapidly damped as the bulk value is approached. The results from theory and simulation agree well, except that the DFT underestimates the behaviour near contact and the contact value itself. The behaviour of $\rho(x)$ away from the contact region is reproduced quite well, with the correct wavelength and decay length of the oscillations. We emphasize that the coverage considered is relatively large (the arrow in figure 1 indicates the jamming coverage).

In figure 1 we also show the density profiles of equilibrium hard rods against a hard wall (i.e. the external potential given in (28)) at the same density. This is obtained from numerical minimization of Percus's functional [10] given in section 3.3. As the hard wall exerts a similar effect to a fixed particle, in the equilibrium case the density profile at the hard wall is equal (up to the bulk density as a multiplicative constant) to the pair correlation function $g(x)$. We have 


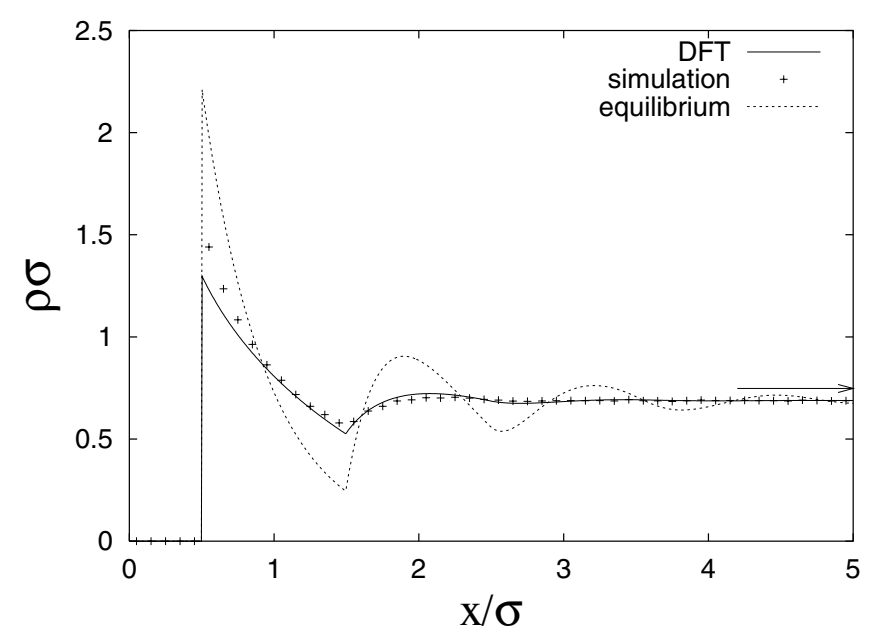

Figure 1. Density profile $\rho(x) \sigma$ as a function of the scaled distance $x / \sigma$ for 1D RSA against a hard wall. The solid curve is the DFT result; the symbols indicate computer simulation data. For comparison, the equilibrium profile of hard rods against a hard wall is also shown (dashed curve). The arrow indicates the density at the jamming limit $\theta=0.7476$.

checked that our result agrees (within reasonable numerical accuracy) with the exact analytic result for the pair distribution function for 1D hard rods [20].

It is striking that in the equilibrium system more structure is built up; the amplitude and decay length of the density profile are considerably larger than in the RSA case. Physically, this means that non-equilibrium in RSA hinders the emergence of structure. Technically, within the DFT approach the origin can be traced back to the fact that the RSA excess free energy functional is smaller (by about a factor $1 / 2$ ) compared to the equilibrium functional. Hence the ideal free energy functional (2) plays a more dominant role than the excess part, which generates the structural correlations. (Note that in the case of adsorption of non-interacting particles, i.e. $F_{\mathrm{exc}}=0$, the density profile is constant for $x>\sigma / 2$.)

As an aside, for RSA in 1D the pair distribution is also known exactly [4]. However, there is a slightly more subtle relation between $\rho(x)$ at the hard wall and $g(x)$. As the hard wall is present from the beginning of the RSA process, it exerts a similar effect to the first particle placed in the bulk case without a wall. To obtain $g(x)$, however, an average over all particle pairs is to be performed; hence this will in general differ from $\rho(x)$.

\subsection{Two dimensions}

Here we apply the 2D functional outlined in section 3.4 to the hard wall represented by the external potential (28), where $x$ is the coordinate perpendicular to the wall. In 2D the jamming limit is known to be $\theta \approx 0.547$ from computer simulations [21]. We consider $\theta=0.4712$ corresponding to $\sim 86 \%$ of the jamming coverage. For the computer simulations we use a wall separation distance $L=11 \sigma$ and periodic boundary conditions in the $y$-direction $(10 \sigma$ perpendicular to the $x$-direction); the number of insertion trials is 1000 . In figure $2 \mathrm{DFT}$ and simulation results are shown. The qualitative behaviour is similar to the result in 1D; however, no kink at $x=3 \sigma / 2$ is present.

Again the agreement of theory and simulation is satisfactory; the only significant deviation is near contact, where the DFT result again underestimates the simulation value. As above in the $1 \mathrm{D}$ investigation, we attribute these deviations to the relatively large coverage considered. 


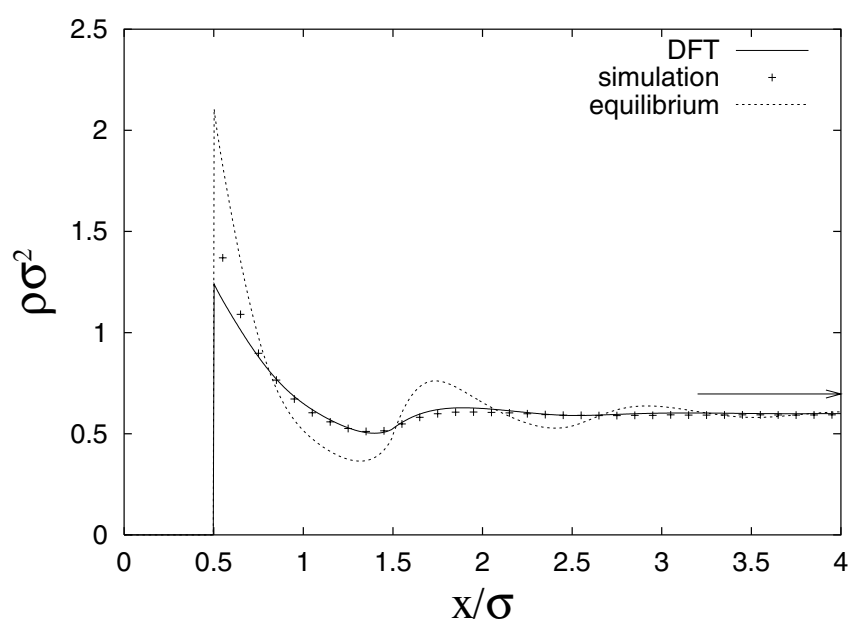

Figure 2. Density profile $\rho(x) \sigma^{2}$ as a function of the scaled distance $x / \sigma$ for 2D RSA against a hard wall. The key is the same as in figure 1 . The arrow indicates the density at the jamming limit $\theta=0.547$

We also present the profile for equilibrium hard discs against a hard wall obtained from numerical minimization of Rosenfeld's 2D functional (section 3.4). Again the equilibrium profile shows significantly stronger structure than the RSA profile.

\section{Conclusions}

In conclusion, we have derived a DFT for the non-equilibrium process of RSA of $d$-dimensional HS onto a $d$-dimensional substrate focusing on the resulting structure not on the adsorption kinetics. To the best of our knowledge, DFT has so far not been applied to RSA. We have explicitly treated in this contribution the space dimensions $d=1,2$; the extension to $d=3$ is straightforward. As was done for other systems before, the exact solution of the 0D limit was used as an input into the powerful machinery of FMT to arrive at explicit approximations for the RSA free energy functional.

Our theory permits the study of spatially inhomogeneous substrates where the adsorption probability at space point $\boldsymbol{r}$ is governed by a Boltzmann weight according to a freely prescribed external potential $V_{\text {ext }}(\boldsymbol{r})$. Such applications include the study of deposition onto stripepatterned substrates [22].

As test applications we focused on the 1D car-parking problem and on 2D colloidal deposition on a solid substrate, and have studied in both cases the effect of an impenetrable boundary region (hard wall) of the adsorption region. When compared to our computer simulation results, we find the theoretical results to correctly describe the oscillatory behaviour with a slight tendency to underestimate the contact value, but with very good agreement of wavelength and decay length of the oscillations into the bulk. The densities in both cases considered are close to the jamming limit; hence a severe test of the accuracy of the DFT is provided. We expect the accuracy of the theoretical curves to further improve upon lowering the density (i.e. moving further away from the jamming limit).

When compared to the corresponding equilibrium systems of hard rods and hard discs against a hard wall, significantly less structured density profiles result at equal bulk densities. We can trace that back to the fact that in RSA the ideal-gas free energy (that smooths out 
structure) plays a more dominant role over the excess free energy (arising from interactions) than in the equilibrium case. This feature was found to be already present in OD. As a rule of thumb, we find the $d$-dimensional excess free energy functional for RSA to be roughly half that of the corresponding equilibrium system.

Hence in conjunction with the machinery of FMT, the OD limit is an, albeit seemingly trivial, useful seed for studying non-trivial behaviour in physically relevant dimensions, and permits one to broaden the scope of DFT to non-equilibrium situations.

\section{References}

[1] Widom B 1966 J. Chem. Phys. 443888

[2] Evans J W 1993 Rev. Mod. Phys. 651281

[3] Schaaf P, Voegel J and Senger B 2000 J. Phys. Chem. B 1042204

[4] Bonnier B, Boyer D and Viot P 1994 J. Phys. A: Math. Gen. 273671

[5] Feder J and Giaever J 1980 J. Colloid Interface Sci. 78144

[6] Feder J 1980 J. Theor. Biol. 87237

[7] Given J A 1992 Phys. Rev. A 45816

[8] Boyer D, Tarjus G and Viot P 1995 J. Chem. Phys. 1031607

[9] Evans R 1992 Fundamentals of Inhomogeneous Fluids ed D Henderson (New York: Dekker) p 85

[10] Percus J K 1976 J. Stat. Phys. 15505

[11] Rosenfeld Y 1989 Phys. Rev. Lett. 63980

[12] Rosenfeld Y, Schmidt M, Löwen H and Tarazona P 1996 J. Phys.: Condens. Matter 8 L577

[13] Rosenfeld Y, Schmidt M, Löwen H and Tarazona P 1997 Phys. Rev. E 554245

[14] Cuesta J A, Martinez-Raton Y and Tarazona P 2002 J. Phys.: Condens. Matter $1411965-80$

[15] Schmidt M 2002 Phys. Rev. E at press

[16] Schmidt M, Schöll-Paschinger E, Köfinger J and Kahl G 2002 J. Phys.: Condens. Matter 14 12099-12117

[17] Schmidt M, Löwen H, Brader J M and Evans R 2002 J. Phys.: Condens. Matter 14 9353-82

[18] Brader J M and Evans R 2002 Physica A 306287

[19] Finken R, Schmidt M and Löwen H 2002 Phys. Rev. E 65016108

[20] Meeron E A and Siegert A J 1968 J. Chem. Phys. 483139

[21] Hinrichsen E L, Feder J and Jossang T 1986 J. Stat. Phys. 44793

[22] Harreis H M, Schmidt M and Löwen H 2002 Phys. Rev. E 65041602 